\title{
Biosystematic studies for some Egyptian Amaranthus L. taxa and their significance in their identification.
}

\author{
Wafaa K. Taia*, Azza A.Shehata, Islam M. El Shamy, Manaser M. Ibrahim
}

Faculty of Science, Botany and Microbiology Department, Alexandria University, Alexandria, Egypt

*Corresponding author: taia55taxonomy@hotmail.com

Abstract

Ten Egyptian Amaranthus species have been collected from the field and their inflorescence, floral characters, pollen grains, fruits, and seeds have been studied carefully by using both stereo- and Scanning electron microscopes. The results has been analyzed using PAST program and gave clustering dendrogram for the studied taxa. The result obtained showed that from the most important character in the identification of the taxa is the number of flowers/unit area of the terminal panicle, beside the type of inflorescence. Pollen morphology gave little account in the circumscription of the studied taxa. Type of fruits dehiscent versus indehiscent fruits can be used in the recognition of some species. Seed shape, micropyle position and microstructure features are more efficient in the identification of the studied taxa beside the fruit characters. In spite of that, seed characters can help in distinguishing the species, but they are not in accordance with the division of the taxa into distinct sections.

Key words: Amaranthus, Bracts, Clustering dendrogram, Fruits, Inflorescence, Pollen grains, Seeds.

\section{Introduction}

Genus Amaranthus L. is considered one of the most important genera belonging to family Amaranthaceae, subfamily Amaranthoideae tribe Amarantheae subtribe Amaranthinae (Muller \& Borsh, 2005). The species within this genus are very widely distributed in the warm and temperate regions. Some of these species are cultivated for its economical use, as grain Amaranth, other ornamentals and some for their edible leaves (Martin \& Ruberte, 1975 and Tanaka, 1976). The species within this genus have been faced with many difficulties in their identifications and their taxonomic divisions since Linnaeus (1753). Linnaeus (1753) divided the genus into two groups; Pentandri and Triandri according to the number of stamens in the flower (which is the same as the number of perianth segments). This division of the genus has been accepted by Adanson (1763) and Tournefort (1794), while Dumortier (1827) reclassified the Amaranthus species into two sections Amaranthotypus and Blitopsis, the former has monoecious plants with pentamerous flowers arranged in lax or dense spikes or panicles and circumsessile fruits, and section Blitopsis which has dioecious plants with trimerous or pentamerous flowers arranged in axillary glomerules and has irregularly ruptured indehiscent capsules.

Gordon (1855) raised the inflorescence type to be a priori character in distinguishing the taxa under the genus and reclassified it into two subgenera; Albersia and Euamaranthus. This division has been accepted by both Kirschleger (1857) and Bentham (1870), but they divided subgenus Albersia into three sections according to the fruit whether bursts transversely or in an irregular manner or indehiscent into Amblogyne, Euamaranthus and Euxolus. Uline (1894) classified the Amaranthus species into four groups; Amblogyne, Scleropus, Pyxidium and Megea according to fruit characters. While Beck (1909) regrouped 


\section{Biosystematic studies for some Egyptian Amaranthus L.}

the Amaranthus species into three sections and Rouy (1910) return back to the old classification of the species into two sections but renamed them Euamaranthus and Pentrius. Formerly, Sauer (1950 \& 1955) classified the genus into two subgenera, differentiating only between monoecious and dioecious species: Acnida (L.) Aellen ex K. R. Robertson and Amaranthus.

In spite of that Thellung (1914), Sauer (1950 \& 1955), Aellen (1959 \& 1972), Cavaco (1962), Zlohary (1966) and Townsend (1973-1979) have been faced with many difficulties in recognizing the different taxa while studying the morphological characters of the genus. The great phenotypic plasticity and hybridization between the individuals of the same species made a great conflict in their identification. Till Mosyakin \& Robertson (1996) the classification of the genus was retained on Dumortier`s (1827) classification who divided it into two sections, Amaranthotypus and Blitopsis. But Mosyakin \& Robertson (1996) found that all the Amaranthus species must be divided into three subgenera; 1- Acnida (L.) Mosyakin \& Robertson (1996) Aellen ex K. R. Robertson with three sections, 2- Albersia (Kunth) Gren. \& Godr. With four sections and 3Amaranthus with three sections and two subsections. All these trials of classification and circumscription of the Amaranthus species, but the taxonomy of the genus is still a matter of controversy.

Floral characters are more stable than vegetative one and more useful in taxonomic decisions. This work has been done to investigate the variations within the floral characters within the Amaranthus species grown in Egypt. According to Täckholm (1976) the genus represented by fourteen annual species growing either as weeds in the cultivated areas or wildly in the road sides everywhere. Ten of these species can be obtained from the field and subjected in this study.

\section{Materials and methods}

Ten Amaranthus species were collected from wild population in Alexandria and Cairo during 2015 and 2016. The ten studied species arranged according to system of infrageneric classification of Mosyakin \& Robertson (1996), their different collection sources are cited in table (1). The collected specimens were identified by the aid of Täckholm (1974) and Boulos (1999). Taxonomic authorities for Latin names and synonymy of the species investigated in this study were based on either Australian plant name index (APNI), Gray card index (GCI) or Index Kewensis (IK). Voucher specimens, was kept in Faculty of Science, Alexandria University, Botany and Microbiology Department.

The Inflorescence type and color have been recorded in the field, while the number of flower per $1 \mathrm{~cm}^{2}$ has been accounted in the lab. The sex of flowers, bracts, bracteoles, tepals, fruit type and seed color were examined using stereomicroscope. The seeds and fruits were examined by SEM, put them onto stubs, covered with gold, and photographed with JEOL JSM-5300 SEM, in Faculty of Science, Alexandria University. The pollen grains have been acetolyzed and described according to Erdtman (1966), for light microscope measurements, 30 pollens/species have been measured and the means are calculated. Non-acetolyzed pollen grains have been sputtered onto SEM stubs, coated with gold for SEM examination. The number of pores was calculated by the following equation: $N=(D / C)^{2}$ Campbell (1992), where $\mathrm{N}$ : number of pores, C: distance between two pores, D: pollen grain diameter. The pore area is calculated according to Toderich et al. (2010). Twenty six floral characters are subjected to clustering analyses using PAST $\mathbf{3}$ programs V3 (Table 7). 


\section{Wafaa K. Taia et al.}

Table 1. Collection data of Amaranthus species and its taxonomic rank after Mosyakin \& Robertson (1996).

\begin{tabular}{|c|c|c|c|c|c|}
\hline Species & Collectors & Locations & Subgenus & Sections & $\begin{array}{l}\text { Sub- } \\
\text { Sections }\end{array}$ \\
\hline A. albus $\mathrm{L}$. & $\begin{array}{l}12 \text { Jan. } 2016 \text {, } \\
\text { by Wafaa Taia }\end{array}$ & $\begin{array}{l}\text { Madinati, } \\
\text { Cairo }\end{array}$ & $\begin{array}{l}\text { Albersia (Kunth) } \\
\text { Gren. \& Godr }\end{array}$ & $\begin{array}{l}\text { Pyxidium } \\
\text { Moquin in DC. }\end{array}$ & -- \\
\hline $\begin{array}{l}\text { A. blitiodes S. } \\
\text { Wats. }\end{array}$ & $\begin{array}{l}11 \text { Aug. 2015, by } \\
\text { Azza Shehata \& } \\
\text { Eslam El-Shamy }\end{array}$ & $\begin{array}{l}\text { Fac. Agric., } \\
\text { El-Shatby, } \\
\text { Alexandria }\end{array}$ & $\begin{array}{l}\text { Albersia (Kunth) } \\
\text { Gren. \& Godr }\end{array}$ & $\begin{array}{l}\text { Pyxidium } \\
\text { Moquin in DC. }\end{array}$ & -- \\
\hline A. caudatus $\mathrm{L}$. & $\begin{array}{l}11 \text { Nov. 2015, by } \\
\text { Wafaa Taia }\end{array}$ & $\begin{array}{l}\text { Madinet } \\
\text { Nasr, Cairo. }\end{array}$ & Amaranthus & Amaranthus & Amaranthus \\
\hline A. graecizans $\mathbf{L}$. & $\begin{array}{l}12 \text { Dec. } 2015 \text {, } \\
\text { by Wafaa Taia }\end{array}$ & $\begin{array}{l}\text { Smouha, } \\
\text { Alexandria }\end{array}$ & $\begin{array}{l}\text { Albersia (Kunth) } \\
\text { Gren. \& Godr }\end{array}$ & $\begin{array}{l}\text { Pyxidium } \\
\text { Moquin in DC. }\end{array}$ & -- \\
\hline A. hybridus L. & $\begin{array}{l}15 \text { Nov. 2015, by } \\
\text { Azza Shehata \& } \\
\text { Eslam El-Shamy }\end{array}$ & $\begin{array}{l}\text { Fac. of Sci., } \\
\text { El-Shatby, } \\
\text { Alexandria }\end{array}$ & Amaranthus & Amaranthus & Hybrida \\
\hline A. lividus $\mathrm{L}$. & $\begin{array}{l}1 \text { Oct. 2015, Azza } \\
\text { Shehata \& Eslam } \\
\text { El-Shamy }\end{array}$ & $\begin{array}{l}\text { Fac. of Sci., } \\
\text { El-Shatby, } \\
\text { Alexandria }\end{array}$ & $\begin{array}{l}\text { Albersia (Kunth) } \\
\text { Gren. \& Godr }\end{array}$ & $\begin{array}{l}\text { Blitopsis } \\
\text { Dumort }\end{array}$ & -- \\
\hline A. retroflexus $\mathrm{L}$. & $\begin{array}{l}14 \text { Oct. 2015, by } \\
\text { Azza Shehata \& } \\
\text { Eslam El-Shamy }\end{array}$ & $\begin{array}{l}\text { Fac. of Sci., } \\
\text { El-Shatby, } \\
\text { Alexandria }\end{array}$ & Amaranthus & Amaranthus & Amaranthus \\
\hline A. spinosus $\mathrm{L}$. & $\begin{array}{l}22 \text { Oct. 2015, by } \\
\text { Azza Shehata \& } \\
\text { Eslam El-Shamy }\end{array}$ & $\begin{array}{l}\text { Al-Agamy, } \\
\text { Alexandria }\end{array}$ & Amaranthus & $\begin{array}{l}\text { Centrusa } \\
\text { Griseb }\end{array}$ & -- \\
\hline A. tricolor $\mathrm{L}$. & $\begin{array}{l}10 \text { Jun. 2015, by } \\
\text { Wafaa Taia }\end{array}$ & $\begin{array}{l}\text { Al-Saha } \\
\text { Square, } \\
\text { Madinet } \\
\text { Nasr, Cairo. }\end{array}$ & $\begin{array}{l}\text { Albersia (Kunth) } \\
\text { Gren. \& Godr }\end{array}$ & $\begin{array}{l}\text { Pyxidium } \\
\text { Moquin in DC. }\end{array}$ & -- \\
\hline A. viridis $\mathrm{L}$. & $\begin{array}{l}1 \text { Oct. 2015, by } \\
\text { Manaser Ibrahim }\end{array}$ & $\begin{array}{l}\text { Smouha, } \\
\text { Alexandria }\end{array}$ & $\begin{array}{l}\text { Albersia (Kunth) } \\
\text { Gren. \& Godr }\end{array}$ & $\begin{array}{l}\text { Blitopsis } \\
\text { Dumort }\end{array}$ & -- \\
\hline
\end{tabular}




\section{Biosystematic studies for some Egyptian Amaranthus L.}

\section{Results}

\section{1-Inflorescence characters (Table 2)}

The inflorescences reddish green or mostly green in color within the studied species, except $A$. blitoides, which is reddish green, A. caudatus, Red, A. blitoides and $A$. tricolor, brown, and A. viridis, brownish green. They are either axillary glomerules only or terminal and axillary glomerules. The length of the terminal panicle varied according the age of the plant; it was $1 \mathrm{~cm}$ in A. lividus and reach $8 \mathrm{~cm}$ in A. caudatus. The axillary glomerules range from $1 \mathrm{~mm}$ to 4 $\mathrm{mm}$. The most important character is the number of flowers / unit area which grouped the studied taxa into three categories:-

1-The number of flowers $/ 1 \mathrm{~cm}^{2}$ less than 25, and gathered A. albus, $A$. graecizans, and $A$. tricolor.

2-The number of flowers / $1 \mathrm{~cm}^{2}$ from 30 to 45, and has A. blitoides, $A$. retroflexus, $A$. spinosus, and $A$. viridis.

3-The number of flowers / $1 \mathrm{~cm}^{2}$ over 75 and has A. caudatus, A. hybridus, and A. lividus.

The inflorescences are straight in all the taxa, except in $A$. caudatus they are pendulous. Male and female flowers are intermingled in both the terminal and axillary glomerules, except in $A$. caudatus and $A$. spinosus the male flowers founded at terminal panicles only while the female flowers allocated in the axillary glomerules only.

\section{2-Bract and bracteoles characters (Table 3)}

One bract subtending each flower with variation of colors ranging from whitish green in $A$. retroflexus, yellowish green in $A$. spinosus, greenish yellow in A. viridis. A. graecizans the bract was brownish yellow, and it was brown in A. tricolor and red in $A$. blitoides and $A$. caudatus and green in the rest of the studied species. The shape of the bract varies from lanceolate to ovate with acute or acuminate apices. In A. spinosus the bract is modified into spine. The bracts may be longer, shorter or equal to the perianth segments. Each bract enclosed two bracteoles which are either foliaceous or hard like spines, while they were membranous in $A$. graecizans only.

\section{3-Flower characters (Table 4)}

The flowers are actinomorphic in all the studied taxa, except $A$. blitoides they are zygomorphic in which the segment parts of the tepals are unequal. The bracteoles differ in color between the taxa, they are white in $A$. retroflexus, membranous with green mid rib in $A$. albus, membranous tinged with pink in A. caudatus, brown in A. tricolor, red in A. caudatus, and green in the rest of the species. The perianth segments are from three to five in both the male and female flowers, with lanceolate to ovate shapes and acute or acuminate apices. The tepals are free in all the species except in A. lividus they are united at the base. The female flowers have one style ended with three stigmas in all the taxa, but in both $A$. tricolor and $A$. viridis two stigmas have been recorded, while in $A$. spinosus four stigmas can be found.

The male flowers have three or five stamens, which may be completely enclosed within the perianth segments or protrude outside the perianth segments or equal in length to the tepals.

\section{4-Fruits and seeds characters (Table 5)}

The fruits in all the taxa are brown or brownish black, except in A. spinosus, the fruits are light brown or brownish yellow. They are elliptic or subglobose dehiscent capsules, except in A. caudatus, A. lividus and $A$. viridis they are indehiscent utricles. The wall of the fruits is smooth or slightly rugate, as seen by the stereomicroscope. The length of mature fruit in relation to the perianth length is mostly equal or slightly longer, except $A$. retroflexus and $A$. spinosus it is equal or shorter than the perianth. In $A$. tricolor the fruit is noticeably shorter than the perianth.

The seeds which are brown, brownish black or black are globose or subglobose, 
Wafaa K. Taia et al.

Table 2. Inflorescence characters within the studies Amaranthus species.

\begin{tabular}{|c|c|c|c|c|c|c|c|c|}
\hline $\begin{array}{l}\text { Character } \\
\text { Species }\end{array}$ & Color & Type & $\begin{array}{l}\text { L. of Ter. Infl. } \\
\text { (cm) }\end{array}$ & $\begin{array}{l}\text { L. of Axil. } \\
\text { Infl. (cm) }\end{array}$ & $\begin{array}{l}\text { Ratio } \\
\text { T/A }\end{array}$ & $\begin{array}{l}\text { flower } \\
/ \mathrm{cm}^{2}\end{array}$ & $\begin{array}{l}\text { Shape of } \\
\text { ter. infl. }\end{array}$ & $\begin{array}{c}\text { Male, female } \\
\text { Flower } \\
\text { position } \\
\end{array}$ \\
\hline A. albus & $\begin{array}{l}\text { Light } \\
\text { green }\end{array}$ & Axil. Glom. & - & $\begin{array}{c}0.1-0.4 \\
(0.27 \pm 0.12)\end{array}$ & - & 22 & Straight & Intermixed \\
\hline A. blitoides & Reddish & Axil. Glom. & - & $\begin{array}{c}0.1-0.15 \\
(0.12 \pm 0.25)\end{array}$ & - & 43 & Straight & Intermixed \\
\hline A. caudatus & Red & $\begin{array}{l}\text { Ter.Pan. \& } \\
\text { Axil. Glom. }\end{array}$ & $\begin{array}{c}1.5-8 \\
(4.75 \pm 3.22)\end{array}$ & $\begin{array}{c}0.9-1.5 \\
(1.12 \pm 0.26)\end{array}$ & 4.24 & 94 & Pendulous & ô ter. \\
\hline A. graecizans & Red & Axil. Glom. & (1) & $\begin{array}{c}0.1-0.2 \\
(0.15 \pm 0.05)\end{array}$ & - & 12 & Straight & Intermixed \\
\hline A. hybridus & Green & $\begin{array}{l}\text { Ter.Pan. \& } \\
\text { Axil. Glom. }\end{array}$ & $\begin{array}{c}2.5-6 \\
(4.17 \pm 1.43)\end{array}$ & $\begin{array}{c}0.2-0.4 \\
(0.308 \pm 0.97)\end{array}$ & 1.35 & 81 & Straight & Intermixed \\
\hline A. lividus & Green & $\begin{array}{l}\text { Ter.Pan \& } \\
\text { Axil. Glom. }\end{array}$ & $\begin{array}{c}1-3 \\
(1.8 \pm 0.57)\end{array}$ & $\begin{array}{c}0.2-0.4 \\
(0.3 \pm 0.1)\end{array}$ & 1.31 & 79 & Straight & Intermixed \\
\hline A. retroflexus & Green & $\begin{array}{l}\text { Ter.Pan \& } \\
\text { Axil. Glom. }\end{array}$ & $\begin{array}{c}5-5.4 \\
(5.2 \pm 0.23)\end{array}$ & $\begin{array}{c}0.1-0.2 \\
(0.15 \pm 0.4)\end{array}$ & 3.46 & 39 & Straight & Intermixed \\
\hline A. spinosus & Green & $\begin{array}{l}\text { Ter.Pan \& } \\
\text { Axil. Glom. }\end{array}$ & $\begin{array}{c}1,3-2 \\
(1.6 \pm 0.29)\end{array}$ & $\begin{array}{c}0,1-0.3 \\
(0.22 \pm 0.09)\end{array}$ & 7.27 & 31 & Straight & $\widehat{\partial}$ ter. $\rho$ axil. \\
\hline A. tricolor & Brown & Axil. Glom. & - & $\begin{array}{c}01-0.15 \\
(0.12 \pm 0.25)\end{array}$ & - & 21 & Straight & Intermixed \\
\hline A. viridis & $\begin{array}{l}\text { Brownish } \\
\text { green }\end{array}$ & $\begin{array}{l}\text { Ter.Pan \& } \\
\text { Axil. Glom. }\end{array}$ & $\begin{array}{c}3-5 \\
(4.6 \pm 0.52)\end{array}$ & $\begin{array}{c}0.2-0.4 \\
(3.4 \pm 1.86)\end{array}$ & 1.35 & 34 & Straight & Intermixed \\
\hline
\end{tabular}

Abbreviations: Axil.=axillary, Glom.=glomerules, Infl.=inflorescence, L.=length, Pan.=panicle, Ter.=terminal, $\mathrm{T} / \mathrm{A}=$ terminal/axillary

Table 3. Bract and bracteole characters of the Amaranthus species.

\begin{tabular}{|c|c|c|c|c|c|c|c|c|}
\hline \multirow[b]{2}{*}{ Characters } & \multicolumn{7}{|c|}{ Bract } & \multirow{2}{*}{$\begin{array}{c}\text { Bracteole } \\
\text { type }\end{array}$} \\
\hline & Color & Shape & $\begin{array}{c}\text { Length } \\
\text { (mm) }\end{array}$ & $\begin{array}{c}\text { Width } \\
\text { (mm) }\end{array}$ & Apex & Modification & $\mathbf{B} / \mathbf{P}$ & \\
\hline A. albus & G & Ov/Lan & $\begin{array}{c}0.29-0.3 \\
(0.29 \pm 0.4)\end{array}$ & $\begin{array}{c}0.11-0.12 \\
(0.11 \pm 0.04)\end{array}$ & Acm & -- & long & Sp \\
\hline A. blitoides & $\mathrm{R}$ & Lan & $\begin{array}{c}0.19-0.2 \\
(0.19 \pm .01)\end{array}$ & $\begin{array}{c}0.1-0.12 \\
(0.1 \pm 0.01)\end{array}$ & Acu & -- & Short & $\mathrm{F}$ \\
\hline A. caudatus & G R & Lan & $\begin{array}{c}0.32-0.4 \\
(0.34 \pm .01)\end{array}$ & $\begin{array}{c}0.13-0.15 \\
(0.14 \pm 0.01)\end{array}$ & Acu & -- & Long & $\mathrm{Sp}$ \\
\hline A. graecizans & By & Lan & $\begin{array}{c}0.2-0.25 \\
(0.23 \pm 0.02)\end{array}$ & $\begin{array}{c}0.11-0.13 \\
(0.12 \pm 0.01)\end{array}$ & Acm & -- & Short & M \\
\hline A. hybridus & G & Lan. & $\begin{array}{c}0.2-0.22 \\
(0.24 \pm 0.01)\end{array}$ & $\begin{array}{c}0.1-0.11 \\
(0.15 \pm 0.02)\end{array}$ & Acm & -- & Long & $\mathrm{Sp}$ \\
\hline A. lividus & G & $\mathrm{Ov}$ & $\begin{array}{c}0.19-0.22 \\
(0.2 \pm 0.01)\end{array}$ & $\begin{array}{c}0.11-0.14 \\
(0.12 \pm 0.01)\end{array}$ & Acu & -- & short & Fol \\
\hline A. retroflexus & WG & Lan. & $\begin{array}{c}0.3-0.5 \\
(0.25 \pm 0.03)\end{array}$ & $\begin{array}{c}0.14-0.19 \\
(0.13 \pm 0.01)\end{array}$ & Acm & -- & Long & $\mathrm{Sp}$ \\
\hline A. spinosus & YG & Ov/Lan & $\begin{array}{c}0.25-0.45 \\
(0.3 \pm 0.09)\end{array}$ & $\begin{array}{c}0.13-0.2 \\
(0.1 \pm 0.01)\end{array}$ & Acm & Into spines & Short & Sp \\
\hline A. tricolor & B & Ov/Lan & $\begin{array}{c}0.38-0.5 \\
(0.42 \pm 0.06)\end{array}$ & $\begin{array}{c}0.19-0.21 \\
(0.2 \pm 0.01)\end{array}$ & Acm & -- & Equal & Sp \\
\hline A. viridis & GY & Ov/Lan & $\begin{array}{c}0.19-0.21 \\
(0.22 \pm 0.01)\end{array}$ & $\begin{array}{c}0.1-0.13 \\
(0.11 \pm 0.01)\end{array}$ & Acm & -- & Short & FM \\
\hline
\end{tabular}

Abbreviations: $\mathrm{Acm}=$ Acuminate, $\mathrm{Acu}=$ Acute, $\mathrm{B} / \mathrm{P}=$ bract/perianth, $\mathrm{B}=$ Brown, $\mathrm{By}=$ Brown yellow, $\mathrm{F}=$ Folacious, $\mathrm{F} M=$ Folacious membranous, $\mathrm{G}=$ green, $\mathrm{GR}=\mathrm{Green}$ tinged with red, $\mathrm{GY}=$ Green tinged with yellow, Lan=lanceolate, $\mathrm{M}=$ Membranous, $\mathrm{Ov}=$ ovate, $\mathrm{R}=\mathrm{Red}, \mathrm{Sp}=$ Spinose, $\mathrm{WG}=$ Whitish green, $\mathrm{YG}=$ Yellowish green 


\section{Biosystematic studies for some Egyptian Amaranthus L.}

Table 4. Floral characters of Amaranthus species

\begin{tabular}{|c|c|c|c|c|c|c|c|c|c|c|c|c|}
\hline \multirow[b]{2}{*}{ Characters } & \multirow[b]{2}{*}{ Sym } & \multicolumn{7}{|c|}{ Perianth Character in both $q$ and ${ }^{\Uparrow}$ flowers } & \multicolumn{3}{|c|}{ No. } & \multirow[b]{2}{*}{$\mathbf{S} / \mathbf{P}$} \\
\hline & & Color & No. & Length (cm) & $\begin{array}{l}\text { Width } \\
\text { (cm) }\end{array}$ & Shape & Apex & State & Sty. & Stig & St & \\
\hline A. albus & A & MG & 3 & $\begin{array}{c}0.19-0.21 \\
(0.2 \pm 0.01)\end{array}$ & $\begin{array}{c}0.1-0.11 \\
(0.11 \pm 0.0)\end{array}$ & Lan & Acm & Free & 1 & 3 & 3 & Lo. \\
\hline A. blitoides & $\mathrm{Z}$ & G & $3-5$ & $\begin{array}{c}0.15-0.18 \\
(0.16 \pm 0.01)\end{array}$ & $\begin{array}{c}0.1-0.19 \\
(0.1 \pm 0.01)\end{array}$ & $\begin{array}{l}\text { Ov/ } \\
\text { Lan }\end{array}$ & Acm & Free & 1 & 3 & 3 & Lo. \\
\hline A. caudatus & A & $\mathrm{R}$ & 5 & $\begin{array}{c}0.25-0.32 \\
(0.28 \pm 0.03)\end{array}$ & $\begin{array}{c}0.15-0.17 \\
(0.16 \pm 0.0)\end{array}$ & Ov & Acm & Free & 1 & 3 & 5 & Sh. \\
\hline $\begin{array}{l}\text { A. } \\
\text { graecizans }\end{array}$ & A & MP & 3 & $\begin{array}{c}0.2-0.29 \\
(0.24 \pm 0.04)\end{array}$ & $\begin{array}{c}0.12-0.15 \\
(0.13 \pm 0.0)\end{array}$ & $\begin{array}{l}\text { Ov/ } \\
\text { Lan }\end{array}$ & $\mathrm{Acu}$ & Free & 1 & 3 & 3 & Sh. \\
\hline A. hybridus & A & G & $3-5$ & $\begin{array}{c}0.2-0.25 \\
(0.21 \pm 0.02)\end{array}$ & $\begin{array}{c}0.1-0.15 \\
(0.12 \pm 0.0)\end{array}$ & $\mathrm{Ov}$ & Acm & Free & 1 & 3 & $\begin{array}{l}3- \\
5\end{array}$ & Eq. \\
\hline A. lividus & A & G & 3 & $\begin{array}{c}0.2-0.25 \\
(0.21 \pm 0.02)\end{array}$ & $\begin{array}{c}0.1-0.5 \\
(0.12 \pm 0.0)\end{array}$ & $\mathrm{Ov}$ & Acu & $\begin{array}{l}\text { unite } \\
\mathrm{d}\end{array}$ & 1 & 3 & 3 & Sh. \\
\hline $\begin{array}{l}\text { A. } \\
\text { retroflexus }\end{array}$ & A & W & 5 & $\begin{array}{c}0.22-0.29 \\
(0.2 \pm 0.01)\end{array}$ & $\begin{array}{c}0.12-0.15 \\
(0.1 \pm 0.01)\end{array}$ & $\begin{array}{l}\text { Ov/ } \\
\text { Lan }\end{array}$ & $\mathrm{C}$ & Free & 1 & 3 & $\begin{array}{l}3- \\
5\end{array}$ & Lo. \\
\hline A. spinosus & A & G & 5 & $\begin{array}{c}0.19-0.21 \\
(0.35 \pm 0.09)\end{array}$ & $\begin{array}{c}0.1-0.12 \\
(0.17 \pm 0.0)\end{array}$ & $\begin{array}{l}\text { Ov/ } \\
\text { Lan }\end{array}$ & Acm & Free & 1 & $3-4$ & 5 & Eq. \\
\hline A. tricolor & A & B & 3 & $\begin{array}{c}0.3-0.5 \\
(0.4 \pm 0.1)\end{array}$ & $\begin{array}{c}0.15-0.19 \\
(0.17 \pm 0.0)\end{array}$ & $\begin{array}{l}\text { Ov/ } \\
\text { Lan }\end{array}$ & Acm & Free & 1 & $2-3$ & 3 & Sh. \\
\hline A. viridis & A & G & 3 & $\begin{array}{c}0.19-0.21 \\
(0.2 \pm 0.01)\end{array}$ & $\begin{array}{c}0.1-0.15 \\
(0.13 \pm 0.0)\end{array}$ & $\mathrm{Ov}$ & $\mathrm{Acu}$ & Free & 1 & $2-3$ & 3 & Sh. \\
\hline
\end{tabular}

Abbreviations: $\mathrm{A}=$ Actinomorphyc, $\mathrm{Acm}=$ Acuminate, Acu= Acute, $\mathrm{B}=$ Brown, $\mathrm{C}=$ Caudate, $\mathrm{Eq} .=$ equal, $\mathrm{G}=\mathrm{Green}$, Lan.=lanceolate, Lo.= longer, $\mathrm{MG}=$ membranous with green midrib, $\mathrm{MP}=$ Membranous tinged with pink, Ov. $=$ ovate, R.=Red, Sh.= shorter, Sty.=style, Stig.=stigma, $\mathrm{St}=$ stamen, $\mathrm{S} / \mathrm{P}=$ stamen/perianth. Sym= symmetry, W=White, $\mathrm{Z}=$ Zygomorphic

Table 5. Fruit and seed characters within the studied Amaranthus species

\begin{tabular}{|c|c|c|c|c|c|c|c|c|c|c|c|}
\hline \multirow{2}{*}{ Character } & \multicolumn{5}{|c|}{ Fruit } & \multicolumn{6}{|c|}{ Seed } \\
\hline & Col & Sh & Ty & Text & $\mathbf{F} / \mathbf{P}$ & Col & Sh & MP & TC & PW & AW \\
\hline A. albus & $\mathrm{BB}$ & $\mathrm{E}$ & DC & $\mathrm{R}$ & Eq. & Bl./BBl. & SG & SF & Elong. & El.S & Sl.Wr \\
\hline A. blitoides & B & $\mathrm{E}$ & DC & S & Eq./L. & Bl. & $\mathrm{Ov}$ & El & Irreg. & GS & Sl.Wr \\
\hline A. caudatus & B & ESG & $\mathrm{U}$ & SR & Eq./L. & $\mathrm{B}$ & G & Ter/El. & Isod. & GS & Pap. \\
\hline A. graecizans & B & SB & DC & R. & L. & $\mathrm{Bl}$ & G. & Sup. & Elong. & Su.Si. & Pun. \\
\hline A. hybridus & B & $\mathrm{SB}$ & DC & SR & Eq./L. & B & G. & Sup. & Isod. & Su.Si & Sm. \\
\hline A. lividus & B & SB & $\mathrm{U}$ & SR & Eq. & DB & G. & Sup. & Isod. & Su.Si & Pun. \\
\hline A. retroflexus & B & $\mathrm{E}$ & DC & SR & Eq./S. & $\mathrm{Bl} / \mathrm{GBl}$ & SG & $\mathrm{La}$ & Isod. & Su.S & Sm. \\
\hline A. spinosus & $\mathrm{BY}$ & $\mathrm{E}$ & DC & $\mathrm{SdRu}$ & Eq./S. & $\mathrm{Bl}$ & G. & Sup. & Hex. & Su.S & Pun. \\
\hline A. tricolor & B & SBE. & Dc. & $\mathrm{R}$ & S. & $\mathrm{Bl} / \mathrm{GBl}$ & G. & Sup. & Elon.P & Su.un & Sm. \\
\hline A. viridis & B & $\mathrm{SB}$ & $\mathrm{U}$ & StR & $\mathrm{L}$. & GBl & G. & Sup. & Isod. & GS & Pun. \\
\hline
\end{tabular}

Abbreviations: $\mathrm{AW}=$ Anticlinal wall, $\mathrm{B}=$ brown, $\mathrm{BB}=$ Blakish brown, , Bl=Black Col=Color, $\mathrm{BBl}=$ Brownish black, $\mathrm{BY}=$ Brown yellow, $\mathrm{DB}=$ Dark brown, $\mathrm{DC}=$ Dehiscent capsule, E=Ellipsoidal, Eq=Equal, El=Ellivated, Elong.= elongated, El.S= elevated straight, ESG=Ellipsoidal to subglobose, $\mathrm{F} / \mathrm{P}=$ Fruit length/ Perianth length, $\mathrm{G}=$ Globose, $\mathrm{GBl}=$ Glossy black, GS= grooved straight, Hex.= hexagonal, Isod.= isodiametric, Irreg.= irregular, $\mathrm{L}=$ Longer, $\mathrm{La}=$ Lateral, $\mathrm{MP}=$ Micropyle position, $\mathrm{Ov}=$ Ovoid, $\mathrm{P}=$ Pentagonal, $\mathrm{Pap}=$ papillate, $\mathrm{Pun} .=$ punctuate, $\mathrm{PW}=\mathrm{Periclinal}$ wall, $\mathrm{S}=$ Smooth, SB=Subglobose, SBE=Subglobose to ellipsoidal, SF= Superficial and forked, SdRu=Smooth downwards and rugose upward, $\mathrm{Sh}=\mathrm{Shape}, \mathrm{Sl}$.Wr= slightly wrinkeled, $\mathrm{Sm} .=$ smooth, $\mathrm{StR}=$ Strongly rugose, $\mathrm{SR}=\mathrm{Smooth}$ to slightly rugose, Sup= Superficial, $\mathrm{SuS}=$ Superficial straight, $\mathrm{Su} . \mathrm{Si}=$ superficial sinuate, $\mathrm{Su} \cdot$ un $=$ superficial undulate, $\mathrm{TC}=\mathrm{Testa}$ cell shape, Ter=Terminal, Tex=Texture, Ty= Type, R= Rugose, $\mathrm{U}=$ Utricle 


\section{Wafaa K. Taia et al.}

except in A. blitoides they are ovoid. The micropyle shape and positions differ between the studied species; they are either superficial in the testa with the same elevations or elevated above the testa. In the second case they are either forked, in A. albus, or take terminal position and crater shape, in $A$. caudatus, or rounded as in the rest of the species.

The outline of the testa cells mostly isodiametric in most of the taxa, while it is elongated in A. albus and A. graecizans with irregular shapes. In $A$. tricolor the outline of the testa cells is elongated pentagonal and in A. spinosus they are hexagonal. Position of the periclinal walls is superficial, grooved or elevated with straight, undulate or sinuate appearance. The anticlinal wall is smooth, punctuate, slightly wrinkeled or papillate (present only in A. caudatus).

\section{5- Pollen grains characters (Table 6)}

The pollen grain of the studied Amaranthus species are a polar, symmetric, spheroidal and pantoporate. They are small, where the radius varied from $16 \mu \mathrm{m}$ in $A$. viridis to $34.4 \mu \mathrm{m}$ in $A$. tricolor. The interporal distance has great variations, even in the same species; it is $4.4 \mu \mathrm{m}$ in both $A$. spinosus and $A$. viridis and reach $9.9 \mu \mathrm{m}$ in $A$. caudatus. Accordingly, the number of pores per pollen varied significantly within the individuals of the same taxa and between the different species as well. The least number of pores was in A. caudatus (30〉pollen), while the maximum number of pores was in $A$. retroflexus, A. spinosus and A. tricolor (over 70 (pollen). The pores, which are moderate in diameter, are superficial or protruded enriched by granules or echinae in different densities. The exine thin, from $1.6 \mu \mathrm{m}$ to 4 $\mu \mathrm{m}$, tectate granulate or echinate with different densities.

\section{6- Clustering analyses of the floral morphological characters (Table 7 \& Dend.)}

Twenty six of the floral characters are subjected to PAST 3 programs V3.as shown in table 7. These characters are either binary, multistate qualitative ordered or multistate qualitative unordered, inflorescence type, and finally multistate quantitative ordered. One character only is continuous, as we avoided the measurable characters to ensure that the subjected ones are taxonomical and not affected by environmental conditions. Also, characters which did not give any significance variations have been excluded. Pollen grains and seeds morphological characters are either hermamegathic changes or environmental ones, and for that they excluded from this analysis. The resulted dendrogram divided the studied taxa into three groups (A, B, C), the group (A) has four species; A. graecizans, A. viridis, A. lividus and $A$. tricolor. The first two species; $A$. graecizans and $A$. viridis; met at Euclidean distance 3.4, while A. lividus and A. tricolor met at Euclidean distance 5.2 and the four species gathered at Euclidean distance 6.2.

The group (B), has five species; $A$. retroflexus, A. hybridus, $A$. blitoides and $A$. albus. The first two species; $A$. retroflexus, $A$. hybridus; met together at Euclidean distance 5.0, while the other two species; A. blitoides and $A$. albus met together at Euclidean distance of 4.8. These four species came together at Euclidean distance 5.3. A. spinosus met the above mentioned four species at Euclidean distance 5.6. The group (C) has one species only, A. caudatus which met with the two previous groups (A \& B) at Euclidean distance 7.4. 
Biosystematic studies for some Egyptian Amaranthus L.

Table 6. Palynological characters within the studied Amaranthus species

\begin{tabular}{|c|c|c|c|c|c|c|c|c|c|c|}
\hline \multirow{2}{*}{ Character } & \multirow{2}{*}{$\begin{array}{l}\text { Pollen } \\
\text { diameter } \\
\text { (D) }\end{array}$} & \multirow{2}{*}{ Chord(C) } & \multirow{2}{*}{ C/D } & \multirow{2}{*}{$\begin{array}{c}\begin{array}{c}\text { No. } \\
\text { of } \\
\text { pores }\end{array} \\
\end{array}$} & \multicolumn{4}{|c|}{ Pore } & \multicolumn{2}{|c|}{ Exine } \\
\hline & & & & & D & El & Or & DG & Thick & Or. \\
\hline A. albus & $\begin{array}{c}18.4-32 \\
(26.08 \pm 4.3 \\
)\end{array}$ & $\begin{array}{c}5.5-7.7 \\
(6.47 \pm 0.85)\end{array}$ & $\begin{array}{c}0.313- \\
0.241\end{array}$ & $36-62$ & $\begin{array}{c}0.25-0.47 \\
(0.38 \pm 0.085)\end{array}$ & Sup. & Gr. & + & $\begin{array}{c}2.4-3.2 \\
(2.72 \pm 0.40)\end{array}$ & Gr. \\
\hline A. blitoides & $\begin{array}{c}17.6-26.4 \\
(22.8 \pm 2.2)\end{array}$ & $\begin{array}{c}5.5-8.8 \\
(6.33 \pm 1.11)\end{array}$ & $\begin{array}{c}0.3125- \\
0.3142\end{array}$ & $36-46$ & $\begin{array}{c}0.5-1 \\
(0.83 \pm 0.288)\end{array}$ & Sl.el & Gr. & +++ & $\begin{array}{c}1.6-3.2 \\
(2.7 \pm 0.57)\end{array}$ & Gr. \\
\hline $\begin{array}{l}\text { A. } \\
\text { caudatus }\end{array}$ & $\begin{array}{c}23.2-28 \\
(25.65 \\
\pm 1.73)\end{array}$ & $\begin{array}{c}5.5-9.9 \\
(5.08 \pm 0.28)\end{array}$ & $\begin{array}{c}0.2371- \\
0.3438\end{array}$ & $30-64$ & $\begin{array}{c}0.3-0.66 \\
(0.48 \pm 0.18)\end{array}$ & Sup. & Gr. & +++ & $\begin{array}{c}1.6-3.2 \\
(2.7 \pm 0.47)\end{array}$ & D.Gr. \\
\hline $\begin{array}{l}\text { A. } \\
\text { graecizans }\end{array}$ & $\begin{array}{c}21.6-31.2 \\
(24.9 \pm 2.7)\end{array}$ & $\begin{array}{c}5.5-8.8 \\
(5.75 \pm 0.96)\end{array}$ & $\begin{array}{c}0.2750- \\
0.2821\end{array}$ & $25-47$ & $\begin{array}{c}0.33-0.9 \\
(0.53 \pm 0.32)\end{array}$ & Sl.el & Gr. & + & $\begin{array}{c}2.4-4 \\
(2.61 \pm 0.47)\end{array}$ & M.Gr. \\
\hline $\begin{array}{l}\text { A. } \\
\text { hybridus }\end{array}$ & $\begin{array}{c}22.4-28 \\
(25 \pm 1.55)\end{array}$ & $\begin{array}{c}5.5-7.7 \\
(5.72 \pm 0.64)\end{array}$ & $\begin{array}{c}0.2371- \\
0.2831\end{array}$ & $44-64$ & $\begin{array}{c}0.9-1.2 \\
(1.06 \pm 0.12)\end{array}$ & Sup. & Gr. & +++ & $\begin{array}{c}1.4-3.2 \\
(2.67 \\
\pm 0.58)\end{array}$ & D.Gr. \\
\hline A. lividus & $\begin{array}{c}18.4-24.8 \\
(21.44 \\
\pm 1.94)\end{array}$ & $\begin{array}{c}5.5-6.6 \\
(5.77 \pm 0.49)\end{array}$ & $\begin{array}{c}0.2865- \\
0.2750\end{array}$ & $43-47$ & $\begin{array}{c}1-1.3 \\
(1.04 \pm 0.075)\end{array}$ & Sup. & Ec & +++ & $\begin{array}{c}2.4-3.2 \\
(2.88 \\
\pm 0.40)\end{array}$ & D.Gr. \\
\hline $\begin{array}{l}\text { A. } \\
\text { retroflexus }\end{array}$ & $\begin{array}{c}24.8-32 \\
(28.38 \\
\pm 2.06)\end{array}$ & $\begin{array}{c}5.5-7.7 \\
(5.533 \pm 0.74)\end{array}$ & $\begin{array}{l}0.2218- \\
0.2406\end{array}$ & $73-62$ & $\begin{array}{c}0.83-1.1 \\
(0.93 \pm 0.085)\end{array}$ & Sup. & Ec & ++ & $\begin{array}{c}1.6-3.2 \\
(2.5 \pm 0.4)\end{array}$ & D.ME \\
\hline $\begin{array}{l}\text { A. } \\
\text { spinosus }\end{array}$ & $\begin{array}{c}16.8-33.6 \\
(27.5 \\
\pm 4.242)\end{array}$ & $\begin{array}{c}4.4-7.7 \\
(5.66 \pm 0.86)\end{array}$ & $\begin{array}{l}0.250- \\
0.2238\end{array}$ & $57-71$ & $\begin{array}{c}0.66-0.83 \\
(0.76 \pm 0.09)\end{array}$ & El. & Ec & +++ & $\begin{array}{c}1.6-2.4 \\
(1.65 \pm 0.2)\end{array}$ & Gr. \\
\hline A. tricolor & $\begin{array}{c}20-34.4 \\
(28.74 \\
\pm 4.4)\end{array}$ & $\begin{array}{c}4.4-7.7 \\
(5.2 \pm 0.94)\end{array}$ & $\begin{array}{l}0.220- \\
0.2238\end{array}$ & $74-71$ & $\begin{array}{c}0.4-0.5 \\
(0.46 \pm 0.057)\end{array}$ & El. & $\mathrm{Sm}$ & -- & $\begin{array}{c}1.6-2.4 \\
(1.94 \\
\pm 0.41)\end{array}$ & D.ME \\
\hline A. viridis & $\begin{array}{c}16-24.8 \\
(22.34 \\
\pm 2.84)\end{array}$ & $\begin{array}{c}4.4-6.6 \\
(5.1 \pm 0.78)\end{array}$ & $\begin{array}{c}0.2750- \\
0.2661\end{array}$ & $47-50$ & $\begin{array}{l}0.9-1.1 \\
(1 \pm 0.1)\end{array}$ & Sl.el & Ec. & + & $\begin{array}{c}1.6-2.4 \\
(2.02 \\
\pm 0.41)\end{array}$ & D.Gr. \\
\hline
\end{tabular}

Abreviations: $\mathrm{D}=$ diameter, $\mathrm{DG}=$ density of granules, D.Gr.=densely granulate, D.ME= densely microechinate, $\mathrm{El}=$ elevation, Gr.=granulate, Ec=echinate, M.Gr= moderate granulate, Orn. $=$ ornamentation, Sup.=superficial, Sl.el= slightly elevated, Thick.= thickness.

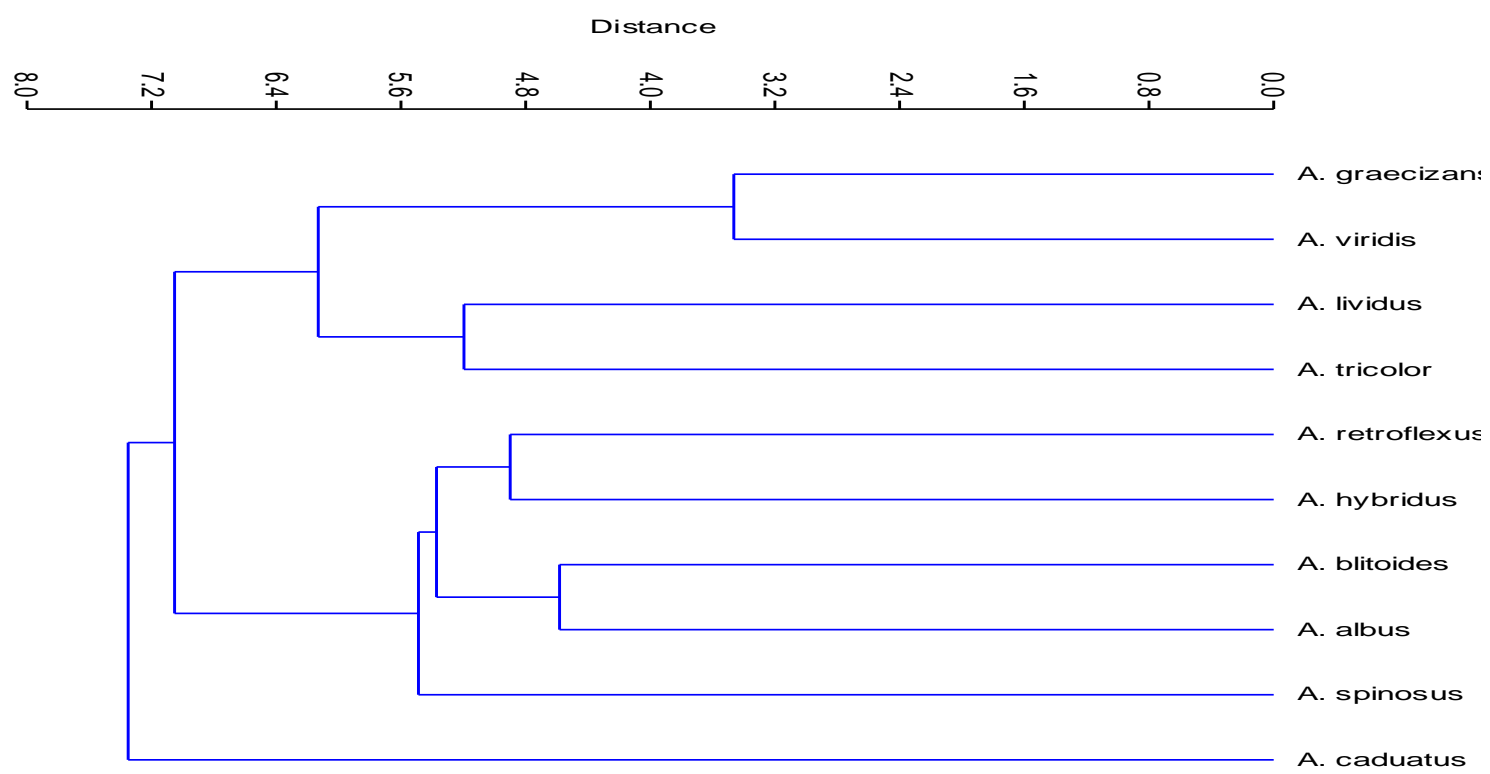

Dendrogram of the floral characters (FMC). 
Table 7. Floral morphological characters (FMC) used in the clustering analyses.

\begin{tabular}{|c|c|c|c|c|c|c|c|c|c|c|c|}
\hline Characters & State & $\begin{array}{c}\text { A. } \\
\text { albus }\end{array}$ & $\begin{array}{l}\text { A. } \\
\text { bli }\end{array}$ & $\begin{array}{c}\text { A. } \\
\text { caudatus }\end{array}$ & $\begin{array}{l}\text { A. } \\
\text { gra }\end{array}$ & $\begin{array}{c}\text { A. } \\
\text { hybridus }\end{array}$ & $\begin{array}{l}\text { A. } \\
\text { lividus }\end{array}$ & $\begin{array}{l}\text { A. } \\
\text { ret }\end{array}$ & $\begin{array}{l}\text { A. } \\
\text { spi }\end{array}$ & $\begin{array}{c}\text { A. } \\
\text { tricolor }\end{array}$ & $\begin{array}{l}A . \\
\text { viridis }\end{array}$ \\
\hline Inflorescence Type & MQl UO & 2 & 2 & 1 & 2 & 1 & 1 & 3 & 3 & 2 & 1 \\
\hline Arrengement & B & 1 & 1 & 2 & 1 & 2 & 2 & 2 & 2 & 1 & 2 \\
\hline Shape & B & 1 & 1 & 2 & 1 & 1 & 1 & 1 & 1 & 1 & 1 \\
\hline Color & M Qn O & 1 & 2 & 5 & 5 & 1 & 1 & 1 & 1 & 4 & 3 \\
\hline Position of flowers & B & 1 & 1 & 2 & 1 & 1 & 1 & 1 & 2 & 1 & 1 \\
\hline †perainth length & B & 1 & 2 & 1 & 1 & 1 & 1 & 1 & 1 & 1 & 1 \\
\hline $\begin{array}{l}\text { Number of } \delta \\
\text { perianth }\end{array}$ & MQnO & 1 & 2 & 4 & 1 & 3 & 1 & 4 & 4 & 1 & 1 \\
\hline $\begin{array}{l}\text { Number of } q \\
\text { perianth }\end{array}$ & MQnO & 1 & 2 & 3 & 1 & 3 & 1 & 3 & 3 & 1 & 1 \\
\hline Perianth state & B & 1 & 1 & 1 & 1 & 1 & 2 & 1 & 1 & 1 & 1 \\
\hline${ }^{\lambda}$ Perianth shape & MQl O & 1 & 2 & 3 & 2 & 3 & 3 & 2 & 2 & 2 & 3 \\
\hline Perianth shape & MQl O & 1 & 2 & 3 & 4 & 4 & 4 & 3 & 1 & 4 & 4 \\
\hline Perianth apex & MQlO & 2 & 2 & 2 & 1 & 2 & 1 & 3 & 2 & 2 & 1 \\
\hline Perianth color & MQl O & 2 & 4 & 5 & 3 & 4 & 4 & 1 & 4 & 6 & 4 \\
\hline No.stigma & MQnO & 3 & 3 & 1 & 3 & 3 & 3 & 3 & 4 & 2 & 2 \\
\hline No.style & $\mathrm{C}$ & 1 & 1 & 3 & 1 & 1 & 1 & 1 & 1 & 1 & 1 \\
\hline No.stamens & MQnO & 1 & 1 & 3 & 1 & 2 & 1 & 2 & 3 & 1 & 1 \\
\hline $\mathrm{S} / \mathbf{P}$ & MQlO & 4 & 4 & 1 & 1 & 3 & 1 & 4 & 2 & 1 & 1 \\
\hline Bracteole type & MQlO & 3 & 2 & 3 & 1 & 3 & 2 & 3 & 3 & 3 & 2 \\
\hline Bract shape & MQlO & 2 & 1 & 1 & 1 & 1 & 3 & 1 & 2 & 2 & 2 \\
\hline Bract apex & B & 2 & 1 & 1 & 2 & 2 & 1 & 2 & 2 & 2 & 2 \\
\hline Bract modification & $\mathrm{B}$ & 1 & 1 & 1 & 1 & 1 & 1 & 1 & 2 & 1 & 1 \\
\hline $\mathbf{B} / \mathbf{P}$ & MQlO & 3 & 1 & 3 & 1 & 3 & 1 & 3 & 1 & 2 & 1 \\
\hline Fruit shape & MQlO & 3 & 3 & 2 & 1 & 1 & 1 & 3 & 3 & 2 & 1 \\
\hline Fruit texture & MQlO & 3 & 1 & 2 & 3 & 2 & 2 & 2 & 2 & 3 & 3 \\
\hline Fruit type & B & 1 & 1 & 2 & 1 & 1 & 1 & 2 & 1 & 2 & 2 \\
\hline $\mathbf{F} / \mathbf{P}$ & MQlO & 4 & 4 & 5 & 6 & 4 & 2 & 3 & 3 & 1 & 6 \\
\hline
\end{tabular}

Abbreviations used: $\mathrm{B} .=$ Binary; $\mathrm{M}$ Ql O=multistate qualitative ordered; $\mathrm{M} . \mathrm{QnO} .=$ multistatequantitave ordered; $\mathrm{M}$ Ql $\mathrm{UO}=$ multistate qualitative ordered; Type of inflorescence: $1=$ panicle, $2=$ glomerules, $3=$ both; Arrangement of inflorescence: 1=axillary, 2=terminal,3=both; Shape of inflorescence: 1 - straight, $2=$ pendulous; Color of inflorescence: $1=$ green, $2=$ greenish red, $3=$ brownish green, $4=$ brown, $5=$ red; Position of $\hat{\delta}$ and + flowers: $1=$ intermixed, $2=\hat{0}$ terminal and + axillary; perainth length: $1=$ equal, $2=$ unequal; Number of $\delta$ perianth: $1=3,2=3-4,3=3-5,4=5$; Number of $q$ perianth: $1=3,2=4-5,3=5$; Perianth state: $1=$ free, $2=$ unitted; $\$$ Perianth shape: $1=$ lanceolate, $2=$ ovate to lanceolate, $3=$ ovate; + Perianth shape: $1=$ linear to lanceolate, $2=$ lanceolate, $3=$ lanceolate to spathulate, $4=$ ovate to lanceolate; Perianth apex: $1=$ acute, $2=$ acuminate, $3=$ codate; Perianth color: $1=$ white, $2=$ membranous with green midrib, $3=$ =membranous with red mid rib, $4=$ green, $5=$ =red, $6=$ brown; Number of stigma: $1=1,2=2-3$, 3=3, 4=3-4; Number of style continuous ( 1 or 3 ); Number of stamens: $1=3,2=3-5,3=5$; Relation between stamens and perianth lengths: $1=$ shorter than, $2=$ as long as, $3=$ as long as or slightly longer, $4=$ longer; Bracteole type: $1=$ =membranous, $2=$ folaceous, $3=$ spinosus; Bract shape: $1=$ lanceolate, $2=$ ovate to lanceolate, $3=$ ovate; Bract apex:1= acute, $2=$ acuminate; Bract modification: 1-no modification, $2=$ spiny bract; Relation between bract/perianth: $1=$ shorter than, $2=$ as long as, $3=$ =longer;fruit shape:1=:subglobose, $2=$ subglobose To Ellipsoidal, $3=$ ellipsoidal; Fruit texture:1= Smooth, $2=$ Smooth to rugose, $3=$ Smooth downwards to rugose upwards,4=Rugose; fruit type: $1=$ dehiscent capsule, $2=$ =indehiscent utricle; Relation between fruit/ perianth: $1=$ shorter than, $2=$ as long as, $3=$ as long as or slightly shorter than, $4=$ as long as or slightly longer than, $5=$ as long as or twice, $6=$ longer than, ret= retroflexus, gra $=$ graecizans, spi $=$ spinosus, bli= blitoides. 
Biosystematic studies for some Egyptian Amaranthus L.
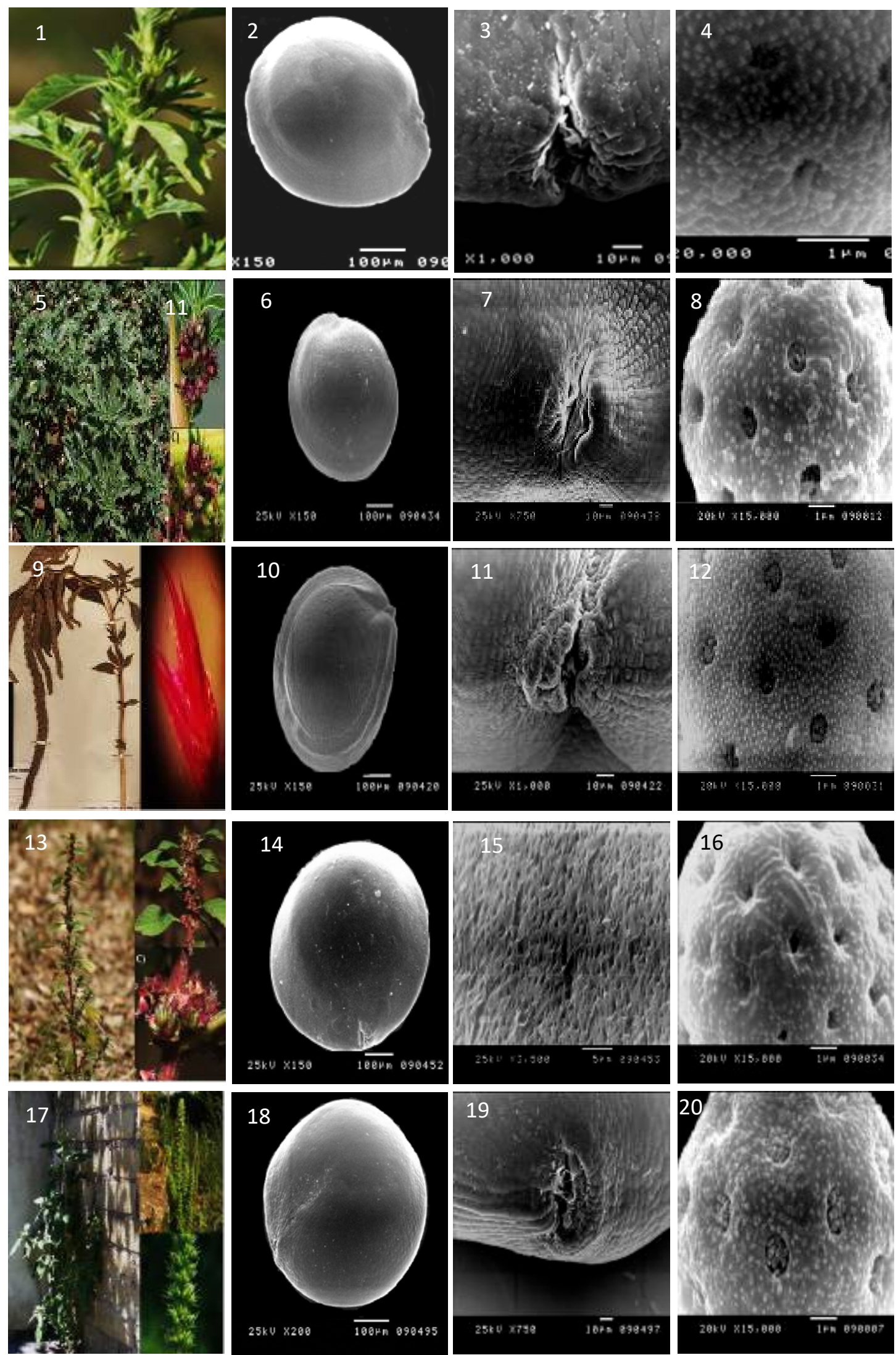

Figs (1-4). A. albus: 1-Whole plant, 2-Seed, 3-Micropyle, 4-Pollen exine (2-4 by SEM)

Figs (5-8). A. blitoides: 5-Whole plant, 6- Seed, 7- Micropyle, 8- Pollen exine (6-8 by SEM)

Figs (9-12). A. caudatus: 9-Whole plant, 10-Seed, 11-Micropyle, 12-Pollen exine (10-12 by SEM)

Figs (13-16). A. graecizans: 13-Whole plant, 14-Seed, 15-Seed surface, 16-Pollen exine (14-16 by SEM)

Figs (17-20). A. hybridus: 17-Whole plant, 18-Seed, 19-Micropyle, 20-Pollen exine (18-20 by SEM) 
Wafaa K. Taia et al.
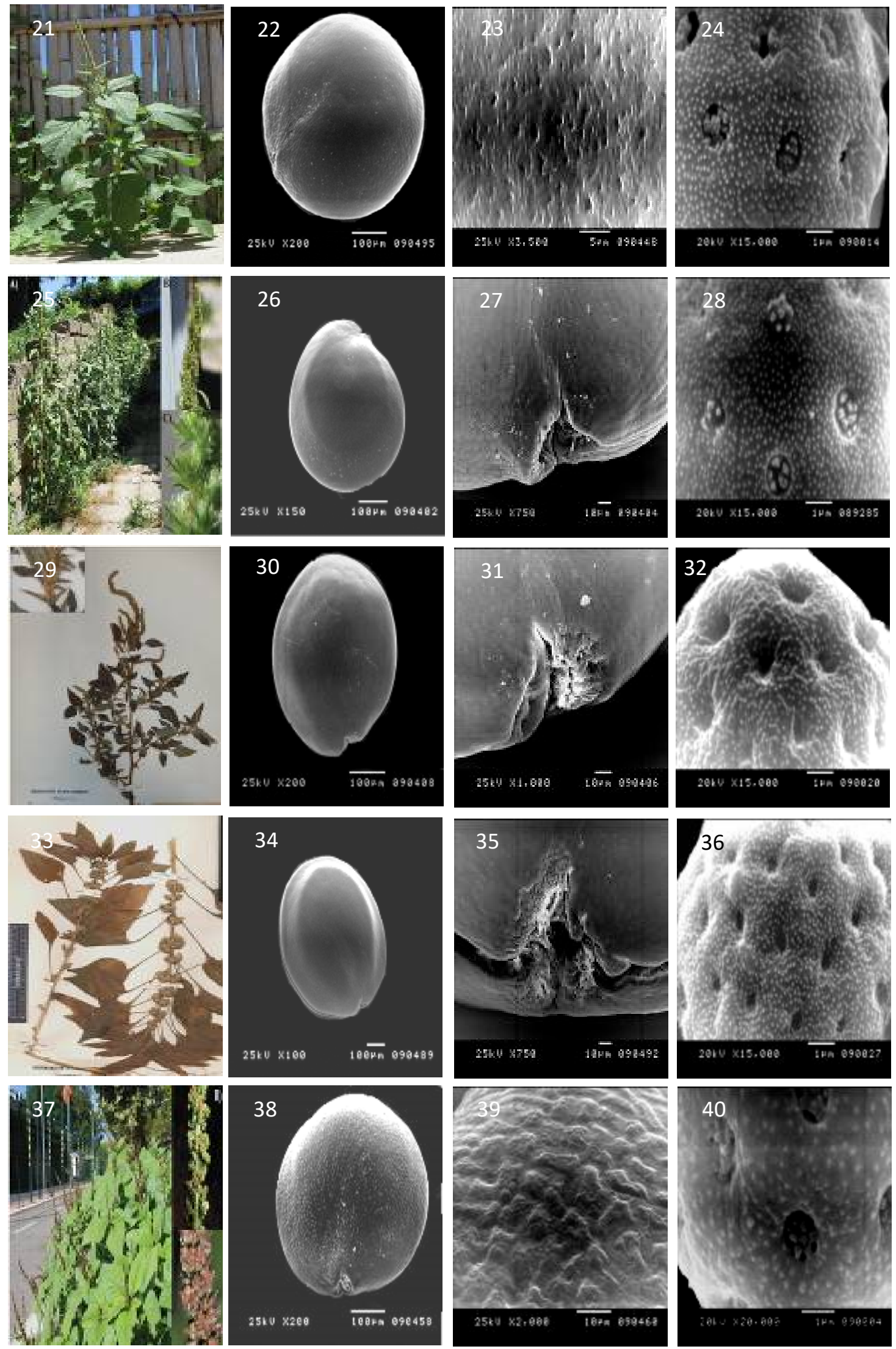

Figs (21-24) A. lividus: 21-Whole plant, 22-Seed, 23-Seed surface, 24-Pollen exine (22-24 by SEM) Figs (25-28) A. retroflexus: 25-Whole plant, 26-Seed, 27-Micropyle, 28-Pollen exine (26-28 by SEM) Figs (29-32) A. spinosus: 29-Whole plant, 30-Seed, 31-Micropyle, 32-Pollen exine (30-32 by SEM) Figs (33-36) A. tricolor: 33-Whole plant, 34-Seed, 35-Micropyle, 36-Pollen exine (30-32 by SEM) Figs (37-40) A. viridis: 37-Whole plant, 38-Seed, 39-Seed surface, 40-Pollen exine (38-40 by SEM) 
Key to the studied species according to their floral variations:

1-All flowers in axillary glomerules

2-Flowers green A. albus

2-Flowers red or brown

3-Bracteoles foliacious........................................... A. blitoides

3-Bracteoles membranous.................................... A. graecizans

3-Bracteoles spinose............................................ A. tricolor

1-Flowers in both axillary glomerules and terminal panicle.

2-Flowers green.

3-Bracteoles foliaceous

A. lividus

3-Bracteoles spinose.

4-Bract modified into spine

A. spinosus

4-Bract not modified

5-Bract white.

A. retroflexus

5-Bract green.

A. hybridus

2-Flowers red or brown.

3-Bracteoles foliaceous.

A. viridis

3-Bracteoles spinose.

A. caudatus

\section{Discussion}

Most of the taxonomical works on genus Amaranthus were classified according to the floral characters and sex of the plants (Mosyakin and Robertson, 2003 and Iamonico, 2009a and 2015). From the time of Linnaeus (1753) who considered the number of stamens as priori character and divided the different Amaranthus species into two groups; Pentandri and Triandri. This division of the genus has been accepted afterwards by Adanson (1763) and Tournefort (1794). Till Dumortier (1827) who considered the sex of the plants are more important in the classification of the genus. While Gordon (1855) considered the inflorescence type to be an important character in distinguishing the taxa under the genus and reclassified it into two subgenera; Albersia and Euamaranthus. This division has been accepted by both Kirschleger (1857) and Bentham (1870), but they divided subgenus Albersia into three sections according to the fruit whether bursts transversely or in an irregular manner or indehiscent into Amblogyne, Euamaranthus and Euxolus. Afterwards Uline (1894) classified the Amaranthus species into four groups; Amblogyne, Scleropus, Pyxidium and Megea according to fruit characters. While
Beck (1909) regrouped the Amaranthus species into three sections and Rouy (1910) return back to the old classification of the species into two sections but renamed them Euamaranthus and Pentrius. Formerly, Sauer (1955) classified the genus into two subgenera, differentiating only between monoecious and dioecious species: Acnida (L.) Aellen ex K. R. Robertson and Amaranthus.

After the quick survey of the history of the classification of the Amaranthus species, we can recognize that floral characters, sex of plant, inflorescence and type of fruits dehiscence are the main characters in the taxonomy of the genus. The data obtained from this investigation divided the studied taxa into three sections, with the exclusion of the sex of plants as all the studied species are monoecious. After the analyses of 26 characters, include inflorescence, bract, perianth segments, and fruits, the clustering dendrogram separate $A$. caudatus in separate section, while the rest of the species divided into two other sections and subsections. A. caudatus is the only species has long, tail like, red pendulous panicle A. graecizans, A. 
viridis, A. lividus, and A. tricolor, in the second section with two subsections, $A$. graecizans, and $A$. viridis in subsection and the other two taxa in the second subsection.

The third group five species; $A$. spinosus, A. blitoides, A. albus, A. hybridus, and $A$. retroflexus; with the separation of $A$. spinosus in a separate subsection, due to its unique bract characters, and gathered both $A$. blitoides, and A. albus in a second subsection and $A$. hybridus and $A$. retroflexus in the third subsection. This division in partial agreement with the previous pioneer taxonomic classifications (Gordon, 1855; Kirschleger, 1857; Bentham, 1870 and Beck, 1909) beside the more recent ones as done by Hauptli and Jain (1978 \& 1984), Joshi and Rana (1991), Mosyakin \& Robertson (1996 \& 2003), Pinto and Velasquez (2010), Iamonico (2012), Iamonico and Das (2014) .

This study reveals that the type of inflorescence, bract state, modification and color, flower color as well as tepal characters can be of use in the circumscription of the studied taxa. This conclusion has been achieved by Costea \& Tardif (2003) who found that bracteoles and the venation in the bracteoles as well as being foliage or membranous within twenty Amaranthus species can be of beneficial use in the circumscription of the genus. As mentioned before (taxonomic status of the genus), all the dioecious species were gathered in subgenus Acnida, while the monoecious ones divided into two subgenera, Amaranthus and Albersia by Mosyakin \& Robertson 1996 which is more acceptable according to our finding. In this study we have to add another important character in the identification of the studied taxa beside the type of inflorescence; panicle versus axillary glomerules; which is the number of flowers within unit length of the inflorescence. This character can differentiate between A. graecizans which has 12 flower $/ 1 \mathrm{~cm}$, while $A$. caudatus, $A$. hybridus and $A$. lividus have from $75-95$ flower $/ 1 \mathrm{~cm}$ and the rest of the studied taxa have moderate number from 20-45 flower/1cm. Meanwhile, we have to consider the type of bracteole as it differentiate $A$. graecizans from the rest of the taxa by having membranous bracteoles, while the rest of the species has either folaceous or spinose bracteoles.

The pollen morphological studies can be of use in many taxa when there will be variations within those taxa. In all the studied Amaranthus species, the pollen grains are apolar, symmetric, spherical and pantoporate. The pollen grains are medium in size and the number of aperture is slightly variable between the studied taxa and between individuals of the same taxa. The number of pores per pollen grains can be used in distinguishing $A$. retroflexus, $A$. spinosus and A. tricolor by having dense pores. Exine, thin tectate with granulate or echinate surfaces. These variations are insignificant and cannot be used in the classification of the genus for being variable as mentioned by Fransen et al. (2001). The pollen characters can be use within higher taxonomic level within the family.

The type of fruits within Amaranthus species considered an important character in the early division of its species as shown by Kirschleger (1857), Bentham (1870), and Uline (1894). The studied taxa have brown, brownish black or brownish yellow capsules. The capsules are either ellipsoidal to subglobose. The dehiscence of the capsules divide the studied taxa into two categories, dehiscent versus indehiscent as mentioned by the above mentioned taxonomists. Fruit shape and type differentiate between the taxa, as $A$. caudatus, A. lividus and $A$. viridis the only ones with utricle fruits while the rest of the species have dehiscent capsules. Barthlott (1984) pointed to the importance of using micro-structural variations in the seed and fruit surfaces in taxonomic studies. Here, the fruit surface did not give significant variations between the species. Seed shape, micropyle position and microstructure features are more efficient in the identification of the studied taxa beside the fruit characters. In spite of that, seed characters can help in distinguishing the 
species, but they are not in accordance with the division of the taxa into distinct sections.

\section{References}

Adanson, M. (1763). Famillies des plantes 11.506.Lehre, paris

Aellen, P. (1959). Amaranthaceae in Hegi, G. (ed.), Illustrierte flora von Mitteleuropa ed. 2, 3(2), 461-532. Carl Hanser Verlag, München.

Aellen, P. (1972). In Rechinger, K. (ed.), Amaranthaceae. Flora Iranica 91: 1-19. Akademische Druck-u.Verlagsanstalt, Austria.

Barthlott, W. (1984). Microstructural Features of Seed Surfaces. In V.H. Heywood and D. M. Moore ed. Current Concept in Plant Taxonomy. Academic Press, London, Orlando. Pp. 95-105.

Beck (1909). Amaranthaceae in Reichenbach, H. G. L. (ed.), Icones florae Germanicae et Helveticae,H.G.Reinchenbach et aliae, Leipzig. 24: 174-177.

Bentham, G. (1870). Flora Australiensis. Vol. 5. (L. Reeve \& Co.: London).

Boulos, L. (1999). Flora of Egypt. Vol.1. (Azollaceae-Oxalidaceae), Al Hadara publishing, Cairo, Egypt.

Campbell, I. D. (1992). .Formula and monogram for estimating the number of regularly patterned elements on the surface of a spheroidal microfossil. Review of Palaeobotany and Palynology. Elsevier Science Publishers B.V., Amsterdam. (72): 165-167.

Costea, M. \& Tardif, F. J. (2003). The bracteoles in Amaranthus (Amaranthaceae) their morphology, structure, function and taxonomic significance. Brit. Org. /Sida 20 (3): 979-985.

Cavaco, A. (1962). Les Amaranthaceae de l'Afrique au sud du tropique du cancer et de Madagascar. Memoires du Museum National $\mathrm{d} \wedge$ Histoire Naturelle, Nouvelle Serie, Serie B, Botanique, 13.

Dumortier, B. C. (1827). Florula Belgica. 19. Acad. Roy. Sci .Letters, Belgique.

Erdtman, G. (1966). Pollen Morphology and Plant Taxonomy: Angiosperms. (An introduction to palynology. I). Hafner Publishing Company, New York (USA).
Fransen, A. S., Skinner, D. Z., Al-Khatib, K. \& Horak, M. J. (2001). Pollen morphological differences in Amaranthus species and interspecific hybrids. Weed Science, 49: 732-737.

Gordon, D. (1855). Florula Juvenalis, 111: 4. Mem. acad. sci., Montpellier.

Hauptli, H. \& Jain, S. (1978). Biosynthetic and agronomic potential of some weedy and cultivated Amaranthus Thor. Appl. Genet., 52: 177-185.

Hauptli, H. \& Jain, S. (1984). Allozyme variation and evolutionary relationships of grain amaranths (Amaranthus spp.) Theor. Appl. Genet., 69: 153-165.

Iamonico, D. (2009a). Il genere Amaranthus L. in Italia: stato attuale delle conoscenze. Annali di Botanica (Roma), Quaderni n. s.

Iamonico, D. (2012). Amaranthus powellii S. Watson subsp. cacciatoi comb. \& stat. nov. (Amaranthaceae). Nord. J. Bot., 30 (1): 1216.

Iamonico, D., (2015). Taxonomic revision of the genus Amaranthus (Amaranthaceae) in Italy. Phytotaxa 199, 1-84

Iamonico, D. \& Das, S. (2014). Amaranthus bengalense (Amaranthaceae) a new species from India, with taxonomical notes on $A$. blitum aggregate Phytotaxa, 181 (5): 293-300.

Kirschleger, Fr. (1857). Flore d'Alsace et de conteres, Litrophes.

Linnaeus, C. (1753). Amaranthus. Pp. 989991 in Species Plantarum, vol. 2. Stockholm: Laurentius Salvius.

Martin, F. W. \& Ruberte, R. M. (1975). Edible leaves of the tropics. Antillien college press, Mayaguez, Puerto Rico. p.17.

Mosyakin, S. L. \& Robertson K. R. (1996). New infra-generic taxa and combinations in Amaranthus L. (Amaranthaceae). Annales Botanici Fennici, 33: 275-281.

Mosyakin, S. L. \& Robertson, K. R. (2003). Amaranthus L. In: Flora of North America Editorial Committee (eds.), Flora of North America North of Mexico (Magnoliophyta: Caryophyllidae) vol. 4, Pp 410-435. Oxford University Press, Oxford.

Müller, K. \& Borsch. T. (2005). Phylogenetics of Amaranthaceae based on 
matK/trnK sequence data: Evidence from parsimony, likelihood, and Bayesian analyses. Annals of the Missouri Botanical Garden, 92: 66-102.

Mueller, F. J. H. von (1859). Amarantaceae. Amarantus pallidiflorus. In: Fragmenta Phytographiae Australiae. Vol. 1(5): 140.

Pinto, G. O. \& Velasquez, W. M. (2010). Sinopsis del subgenero Amaranthus (Amaranthus, Amaranthaceae) en Venezuela. Acta Bot. Venezuelanica, 33: 329-356.

Rouy, G. C. C. (1910). Flora of France x11: 21. Societê des science naturelles de la Charente-inferieure, Paris.

Sauer, J. D. (1950). The grain amaranthus: Asurvey of their history and classification. Annals of the Missouri Botanical Garden. 37: 561-626.

Sauer, J. D. (1955). Revision of the dioecious amaranths. Madrono, 13: 5-46.

Täckholm, V. (1976). Students' Flora of Egypt (ed.2): Cairo University.

Tanaka, T. (1976). Tanka's cyclopedia of edible plants of the world. Keigata publishing company, Tokyo, Japan.
Thellung, A. (1914). Amaranthus. In p. Ascher son and P. Graeber (eds.), Synopsis der Mitteleuropaischen flora 5(1): 225-356. Verleg Von Gebruder Borntraeger, Leipzig.

Toderich, K. N.; Shuyskaya, E. V.; Ozturk, M.; Juylova, A. \& Gismatulina, L. (2010). Pollen morphology of some Asiatic species of genus Salsola (chenopodiaceae) and its taxonomic relationships. Pak. J. Bot., Special Issue (S. I. Ali Festschrift) 42: 155-174.

Tournefort T. P. (1794). Institutiones rei herbariae, Editio Altera, Paris.

Townsend, C. C. (1973). Notes on Amarnthaceae 1. Kew Bull. 28: 141-146.

Townsend, C. C. (1979). Notes in Amaranthaceae 4. kew Bull. 33: 417-419.

Uline, E. B. (1894). A preliminary synopsis of the North American species of Amaranthus. Bot. Gaz. 19: 267-272 and 313320.

Zohary, M. (1966). Flora Palaestina. 1: 180188. Israel Academy of science and Humanities, Jerusalem. 